\title{
Measuring Chamber Pressures of Different Caliber Artilleries Using a Capacitive Pressure Sensor
}

\author{
Xin'e $\mathrm{Li}^{1,2}$, Jing $\mathrm{Zu}^{1,2}$, Tiehua $\mathrm{Ma}^{1,2}$ and Peng $\mathrm{Xu}^{2}$ \\ ${ }^{1}$ Science and Technology on Electronic Test \& Measurement Laboratory, Taiyuan 030051, China \\ ${ }^{2}$ North University of China, Taiyuan 030051, China
}

(Received April 14, 2010; accepted September 8, 2010)

Key words: capacitive pressure sensor, piezo gauge shell, applications, measurement

In order to measure the chamber pressures of different caliber artilleries, a novel capacitive pressure sensor with an elastic pressure-sensitive element is presented in this paper. It uses a circuit cylinder as its stationary anode and the internal electronic piezo gauge shell as its moveable cathode with an electrode distance of $0.5 \mathrm{~mm}$, and constructs a coaxial cylinder capacitor converter. The device principle and structure are given and the device mechanical and thermal characteristics are theoretically simulated using the finite element software ANSYS. Various physical properties were experimentally tested. Results show that the device possesses a very good temperature stability and its capacitance shows an approximately linear relationship with the subjected pressure. It has a sensitivity of $0.00753 \mathrm{Pf} / \mathrm{MPa}$, and a pressure measurement range of 0-600 MPa, and can withstand an instantaneous high temperature of $3,000^{\circ} \mathrm{C}$. By using the sensor shell as the device elastic sensitive element, its volume is reduced and its applications are extended; therefore, it can be used to measure the chamber pressures of various different caliber guns. In addition, its lower cost is helpful in promoting wide applications.

\section{Introduction}

Chamber pressure is defined as the force per unit area that explosive gas exerts on the wall of a gun chamber when a gun is fired. As an important parameter of the artillery system, it is one of the characteristic parameters that must be tested and verified during research and development or product acceptance. The two types of apparatus used to measure it are the internal copper post (copper ball) piezo gauge and internal electronic piezo gauge (IEPG). ${ }^{(1)}$ The former was invented one century ago. It is directly put in the gun chamber and is capable of obtaining the maximum pressure by detecting only its distortion quantity. Although convenient, it is incapable of recording the dynamic change of the chamber pressure with time. (2) It has been gradually replaced by the latter that can be conveniently and accurately used to plot a pressure-time curve.

*Corresponding author: e-mail: Zn1lxe@163.com 
Currently, an IEPG produced in China, as shown in Fig. 1, adopts an imported piezoelectric pressure sensor, which is too large for the chamber pressure measurement of smaller caliber guns. Figure 2 shows the third-generation B251 IEPG produced by the Austrian HPI Corporation in 2000. Similarly to the internal copper post (copper ball) piezo gauge, with a smaller volume of $22 \mathrm{~cm} 3$ and a suitable measurement range of 0 $600 \mathrm{MPa}$, it meets the requirements of chamber pressure measurement for middle and small caliber guns. However, it is too expensive for widespread use.

In this paper, a new type of the so-called capacitive pressure sensor (CPS) based on the above-mentioned IEPG is reported. A schematic of the IEPG application to test the chamber pressure is shown in Fig. 3. IEPG together with the dynamite canister is set in the breech of the gun chamber. When the gun is fired, the pressure caused by the explosion in the chamber is assumed to uniformly act on the surface of the piezo gauge. The plastic deformation of the lead piece inserted into the center of the IEPG along the axis shows that the IEPG shell may experience a greater elastic deformation during explosion. ${ }^{(3)}$ Therefore, the design idea of CPS is inspired, with the shell of such a sensor as the elastic pressure-sensitive element of CPS. Compared with IEPG, CPS has a higher sensitivity, a lower temperature drift, and a lower power consumption. ${ }^{(4,5)}$ The sensor will significantly reduce the size of the piezo gauge, and lower IEPG cost. Moreover, it can be used to measure the chamber pressures of guns with large, medium, and small calibers. CPS will provide great technical support for the development, experimentation, acceptance and production of guns, cartridges, missiles, rockets and fuses among others.

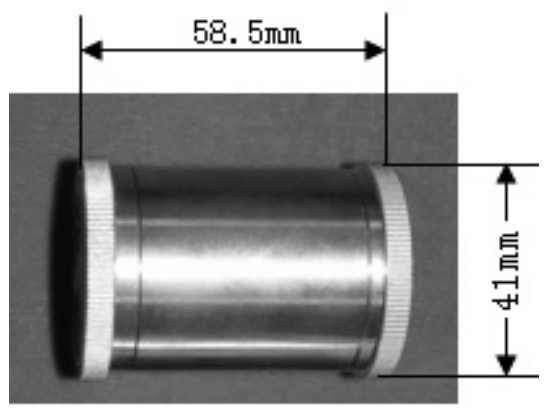

Fig. 1. IEPG made in China.

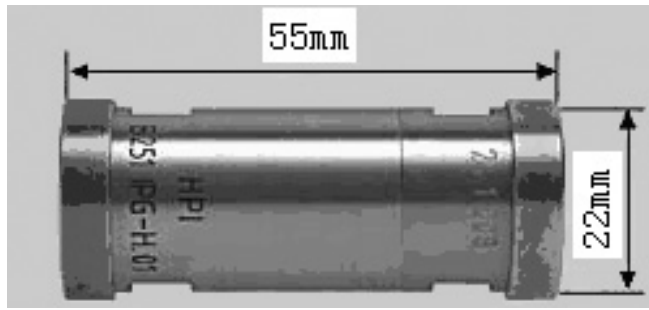

Fig. 2. IEPG made by Austrian HPI Corporation. 


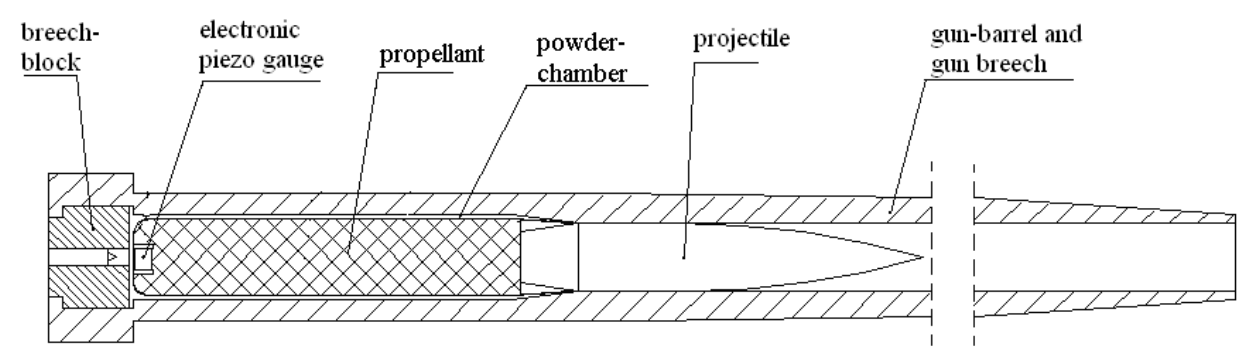

Fig. 3. Schematic of IEPG application to test chamber pressure.

\section{CPS Structure and Principle}

IEPG mainly consists of the outer shell, circuit tube, and end covers. Its circuit tube and outer shell constitute a coaxial cylinder. Since the IEPG outer shell is a very good elastic and pressure-sensitive element, it can be used as the pressure measuring component of CPS. ${ }^{(6,7)}$ In the form of the coaxial cylinder, CPS's two electrodes consist of the outer shell, as a grounded, elastically vibrating cathode under the explosive pressure, and the circuit tube, as a stationary anode. The CPS structure is shown in Fig. 4, where the locating ring is used to ensure coaxiality of the two cylinders. The insulation pad assures good insulation between the outer shell and the circuit tube.

The physical model of the coaxial cylinder is shown in Fig. 5. The internal radius of the outer shell is $R_{\mathrm{A}}$, the outer radius of the circuit tube is $R_{\mathrm{B}}$, and the length is $L$, When $L » R_{\mathrm{A}}-R_{\mathrm{B}}$, the cylinder can approximately be thought of as infinitely long, and the edge effect can be ignored. If $\eta$ is the electric quantity of the inner cylinder unit length, and the electric field strength between the two cylinder $E$ is

$$
E=\frac{\eta}{2 \pi \varepsilon_{0} r}
$$

then the electric potential difference is:

$$
U_{\mathrm{AB}}=\int_{R_{\mathrm{A}}}^{R_{\mathrm{B}}} \frac{\eta}{2 \pi \varepsilon_{0} r} \mathrm{~d} r=\frac{\eta}{2 \pi \varepsilon_{0}} \ln \frac{R_{\mathrm{B}}}{R_{\mathrm{A}}} .
$$

Because each electrode's electric quantity $q=\eta L$, the capacity is

$$
C=\frac{q}{U_{\mathrm{AB}}}=\frac{2 \pi \varepsilon_{0} L}{\ln \frac{R_{\mathrm{B}}}{R_{\mathrm{A}}}},
$$

where $\varepsilon_{0}$ is the vacuum dielectric constant.

When the stress and strain caused under the explosive pressure act on the outer electrode and make it elastically deformed, changes in $R_{\mathrm{B}}$ lead to changes in 


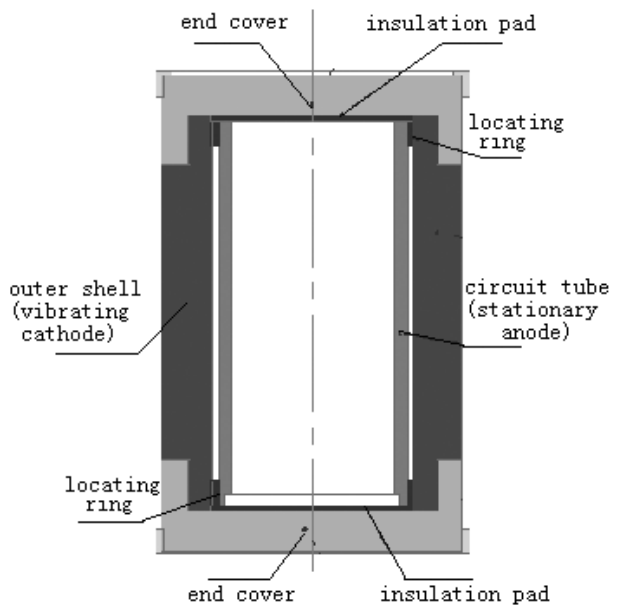

Fig. 4. Schematic structure of CPS.

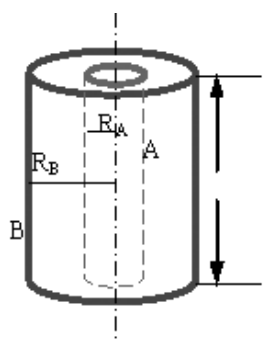

(a)

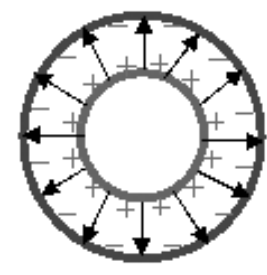

(b)

Fig. 5. Basic structure of the coaxial cylinder.

capacitance..$^{(1,4,8,10)}$ The inside and outside plates are placed asymmetrically, namely, the inside plate is on top of the outer plate, and the outside electrode is the circuit common ground. This structure can effectively supress environmental sensing effect.

\section{CPS Design and Analysis}

\subsection{Analysis of elasticity and plasticity of CPS outer shell}

The CPS outer shell mechanical model is shown in Fig. 6. The deformation of the CPS outer shell under pressure can be simplified to the strain produced by pressure on the elastically deformed plane of a thick cylinder. In elasticity, the radial, angular, and axial stress components are shown as follows, respectively, ${ }^{(11,12)}$

$$
\sigma_{\mathrm{r}}=-p_{\mathrm{e}} \frac{b^{2}}{b^{2}-a^{2}}\left(1-\frac{a^{2}}{r^{2}}\right)=\sigma_{3},
$$




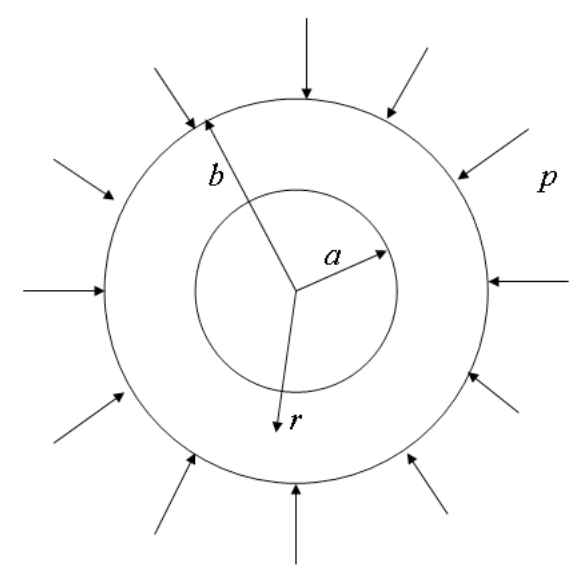

Fig. 6. Mechanical model.

$$
\begin{gathered}
\sigma_{\theta}=-p_{\mathrm{e}} \frac{b^{2}}{b^{2}-a^{2}}\left(1+\frac{a^{2}}{r^{2}}\right)=\sigma_{1}, \\
\sigma_{z}=-p_{\mathrm{e}} \frac{b^{2}}{b^{2}-a^{2}}=\frac{\sigma_{\mathrm{r}}+\sigma_{\theta}}{2}=\sigma_{2},
\end{gathered}
$$

where $a$ and $b$ are the inner and outer radii of the CPS outer shell, respectively, and $p_{\mathrm{e}}$ is the elastic pressure limit. When the pressure on the inner surface of the inner cylinder is zero, the deformation $\Delta d$ of the inner wall along the radial direction is

$$
\Delta d=\frac{2 p_{\mathrm{e}} a b^{2}}{b^{2}-a^{2}} \frac{1}{E}
$$

where $E$ is the elastic modulus of the outer shell material.

According to the sensor's characteristics, the outer shell of the pressure sensor works in the range of its elasticity, the elastic stress $\sigma_{i}$ is given as follows:

$$
\sigma_{i}=\frac{1}{\sqrt{2}} \sqrt{\left(\sigma_{1}-\sigma_{2}\right)^{2}+\left(\sigma_{2}-\sigma_{3}\right)^{2}+\left(\sigma_{3}-\sigma_{1}\right)^{2}}
$$

When the outer pressure reaches the maximum, or elastic pressure limit $p_{\mathrm{e}}$, the points on the inner wall of the shell yield. From von-Mises yield condition, when $\sigma_{i}=\sigma_{\mathrm{S}}$, the corresponding outer pressure $p_{\mathrm{e}}$ is obtained as follows:

$$
p_{\mathrm{e}}=\left(1-\frac{a^{2}}{b^{2}}\right) \frac{\sigma_{\mathrm{S}}}{\sqrt{3}}
$$

where $\sigma_{\mathrm{S}}$ is the yield limit. 
Depending on the size of the circuit board, the outer diameter of the circuit tube as the anode is $16 \mathrm{~mm}$, and the wall thickness is $0.5 \mathrm{~mm}$. The inner diameter of the CPS outer shell as the cathode is taken as $a=17 \mathrm{~mm}$. Therefore, the clearance of two coaxial cylinders or the capacitor spacing is $0.5 \mathrm{~mm}$. The CPS outer shell and end cover material is chosen as $18 \mathrm{Ni}$ maraging steel. Its yield limit $\sigma_{\mathrm{S}}=2,100 \mathrm{MPa}$, elastic modulus $E=200 \mathrm{GPa}$, and Poisson ratio $\mu=0.3$. According to eq. (9), when external pressure $p_{\mathrm{e}}=600 \mathrm{MPa}$, the calculated minimum outer diameter of the CPS outer shell is $b=24.5 \mathrm{~mm}$. For safety reasons, $b$ is taken as $26 \mathrm{~mm}$. The thickness of the thermal barrier layers attached to two ends of the chamber body is $0.5 \mathrm{~mm}$, and the external diameter of the CPS outer shell is $27 \mathrm{~mm}$. The height of the sensor outer shell is $25 \mathrm{~mm}$ and the thicknesses of the end covers are $6 \mathrm{~mm}$. The total height of CPS is $h=37 \mathrm{~mm}$; therefore, the total volume is $V=21 \mathrm{~cm}^{3}$. Thus, under a $600 \mathrm{MPa}$ pressure, the external chamber still operates in the range of elastic deformation. ${ }^{(5)}$ The outer shell and end cover geometric structure is shown in Fig. 7.

\subsection{Analysis of the stress distribution on CPS ends}

Figure 8 shows the actually measured chamber pressure curve. With such a strong pressure exerted on the outside of the shell, a finite element model is set up and used to analyze the stress distribution on it. To simplify analysis and shorten computing time, the circular ends and cylindrical surface of CPS are separately considered. The axissymmetric model is established to analyze the intensity of the end cover, as shown in Fig. 9.(13) By the symmetry on A lines in Fig. 9, the node along the $x$-direction displacement is limited to 0 , on B lines in Fig. 9, the node along the $y$-direction displacement is limited to 0 . Under the $600 \mathrm{MPa}$ pressure applied, the stress on the end cover is smaller than the yield stress of 2,100 MPa; therefore, the end cover is safe.

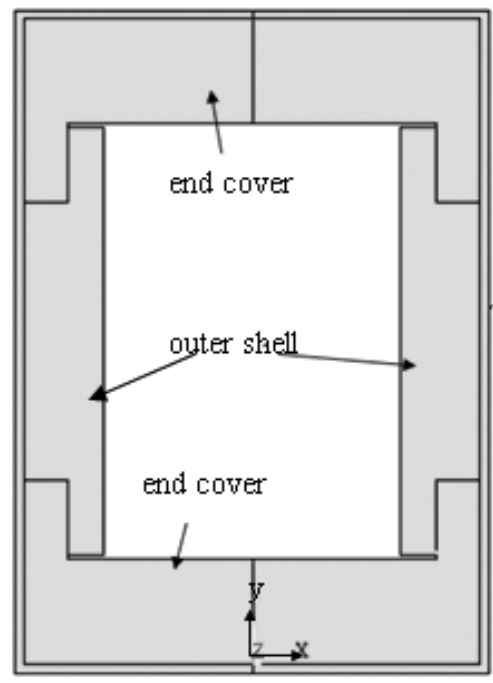

Fig. 7. Geometric structure of the outer shell and end covers. 


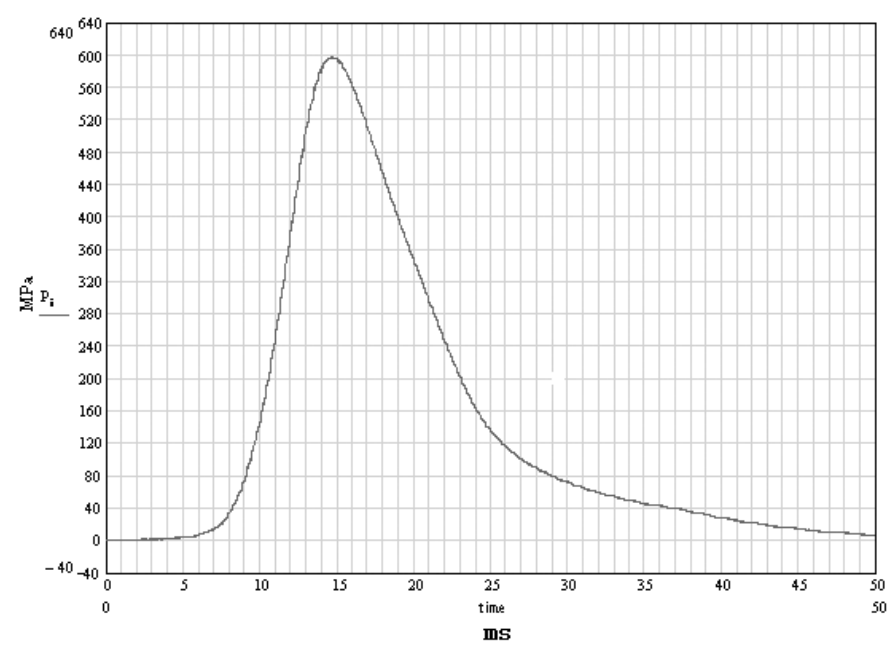

Fig. 8. Measured chamber pressure curve.

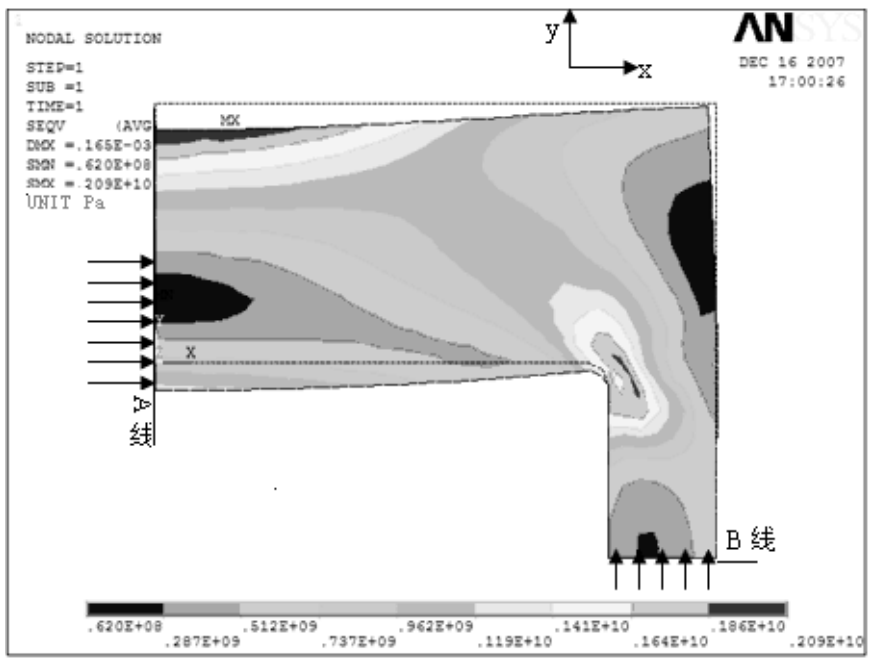

Fig. 9. Von-Mises equivalent stress distribution $(\mathrm{Pa})$ on the end cover.

\subsection{Analysis of stress distribution around the sensor outside}

The outer shell displacement of CPS under the external pressure is obtained using the simulation program ANSYS. In the simulation, based on the cylindrical symmetry of pressure in the gun chamber, the CPS shell is compsed of concentric rings with inner and outer diameters of 17 and $26 \mathrm{~mm}$, respectively. The axisymmetric model of the cylindrical shell was established, namely, the geometric model shown in Fig. 10. $p_{\mathrm{e}}$ is 


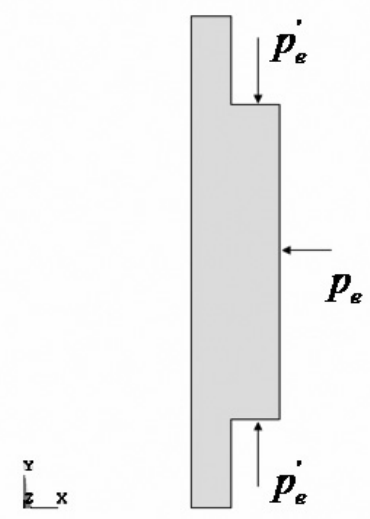

Fig. 10. Geometric model of the outer shell.

the external pressure $\left(p_{\mathrm{e}}=600 \mathrm{MPa}\right), p_{\mathrm{e}}^{\prime}\left(p^{\prime}{ }_{\mathrm{e}}=1,469 \mathrm{MPa}\right)$ is the pressure that acts on the circle area, namely, external pressure is transmitted to the circle area through the end cover. The pressure value considers the ratio of the compression area of the end cover upper rear and the action area of the circle. The pressure curve used in the analysis is partly cut from Fig. 6. The time corresponding to the maximum pressure $600 \mathrm{MPa}$ is $9.5 \mathrm{~ms}$. Figure 11 shows the Von-Mises equivalent stress distribution of the outer shell at a time of $9.5 \mathrm{~ms}$. Figure 12 gives the distortion of the outer shell along the radial direction.

\subsection{Analysis of dynamic characteristics}

The dynamic characteristics of the elastic sensitive component and the hysteresis during transformation are related to the CPS inherent vibration frequency. Generally, the higher the frequency, the better the CPS. It can be calculated through the ANSYS simulation. The inherent frequency of the first-order vibration mode of the outer shell as the sensitive component is $52.665 \mathrm{kHz}$, as shown in Fig. 13, which is much higher than the chamber pressure frequency, $2 \mathrm{kHz}$. Therefore, compared with that of the cylinder, the chamber pressure can be considered as the quasi-static load. Under the chamber pressure, the CPS shell will tend to distort inward.

Figure 14 is a quarter model of the CPS outer shell, The distortion cases in dot1 and dot 2 along the $x$ - and $y$-directions are shown in Figs. 15 and 16, respectively. The distortion size and the directions of the two points are exactly the same, so the chamber pressure load does not cause the first-order vibration mode of the outer shell.

\subsection{CPS temperature effect}

Because the outer wall of CPS directly touches the high temperature gas, the thermal barrier coating (TBC) is added to protect the shell against its thermal deformity, which leads to changes in characteristics and to measurement errors. TBC is a double-layer 


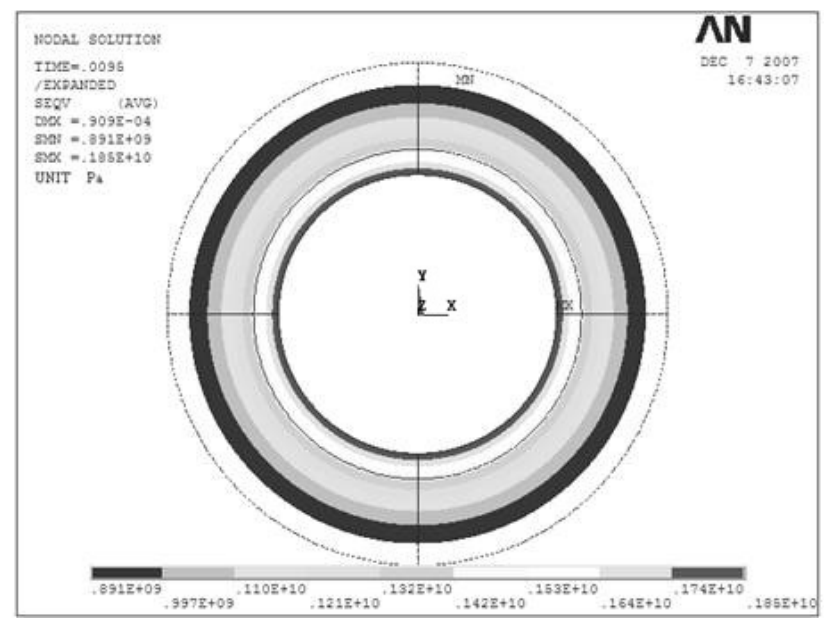

Fig. 11. Von-Mises equivalent stress $(\mathrm{Pa})$ on the outer shell at $9.5 \mathrm{~ms}$.

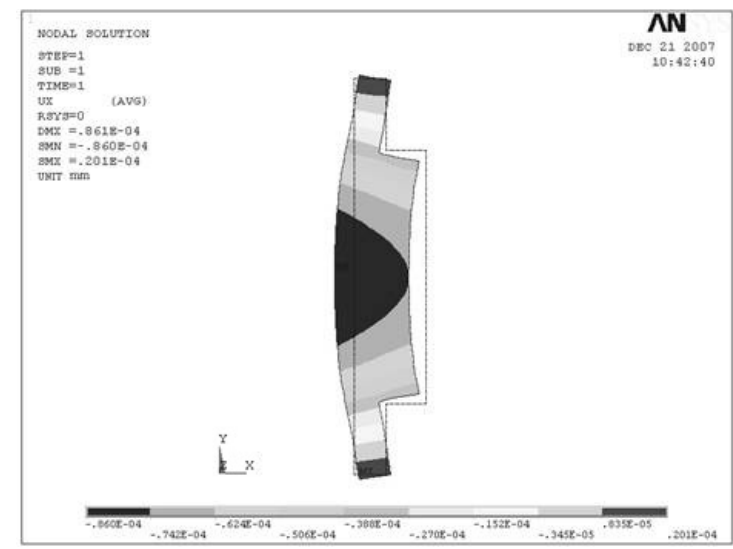

Fig. 12. Distortion ( $\mathrm{mm})$ of outer shell along the radial direction.

structure, with a heat-insulating ceramic as its surface layer and an anti-gasified tack coat as its second layer. The first layer is usually $250-300 \mu \mathrm{m}$ thick and the second layer is approximately $120-150 \mu \mathrm{m}$ thick, and the total thickness is approximately $450 \mu \mathrm{m}$. The heat-insulating coefficient is about 0.05 .

As shown in Figs. 17-20, the temperature characteristics of the shell with TBC are analyzed through ANSYS. Supposing that the initial temperature is $20^{\circ} \mathrm{C}$, the pressure exerted on the outside of the shell reaches maximum about $9.5 \mathrm{~ms}$ after explosion, at which time the temperature load on the surface is $3,000^{\circ} \mathrm{C}$. As shown in Fig. 17, by conservative analysis, namely, by using the first kind of boundary condition of the 


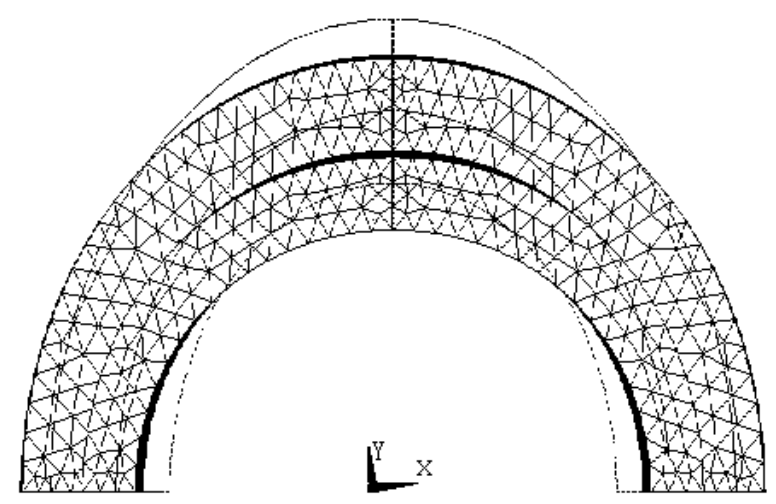

Fig. 13. First-order vibration mode of the tube body.

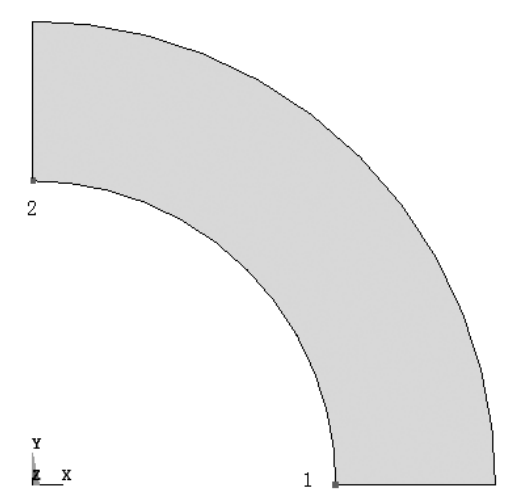

Fig.14. Quarter model of the outer shell.
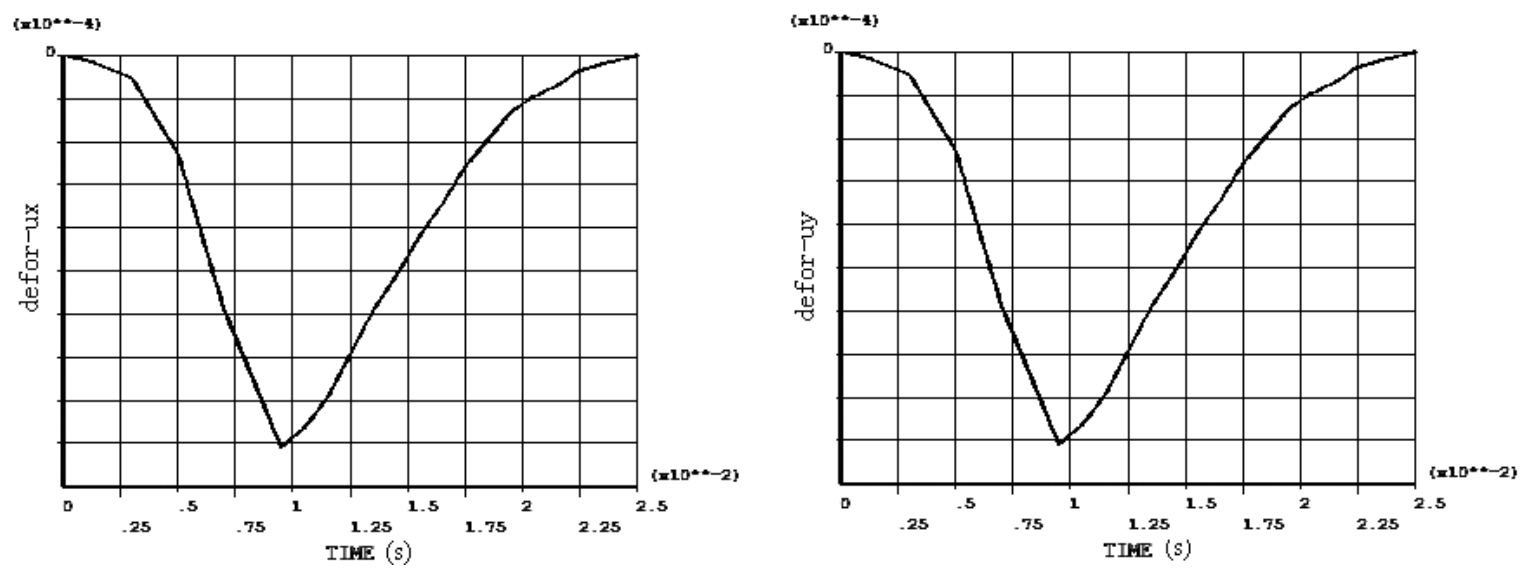

Fig. 15. (left). Distortion of dot 1 along $x$-direction.

Fig. 16. (right). Distortion of dot 2 along $y$-direction. 


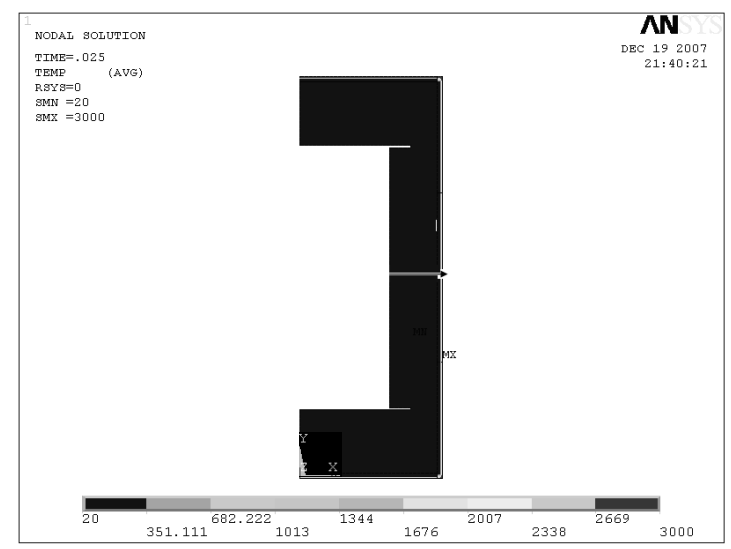

Fig. 17. Temperature distribution on the shell at $3,000^{\circ} \mathrm{C}$ at $25 \mathrm{~ms}$.

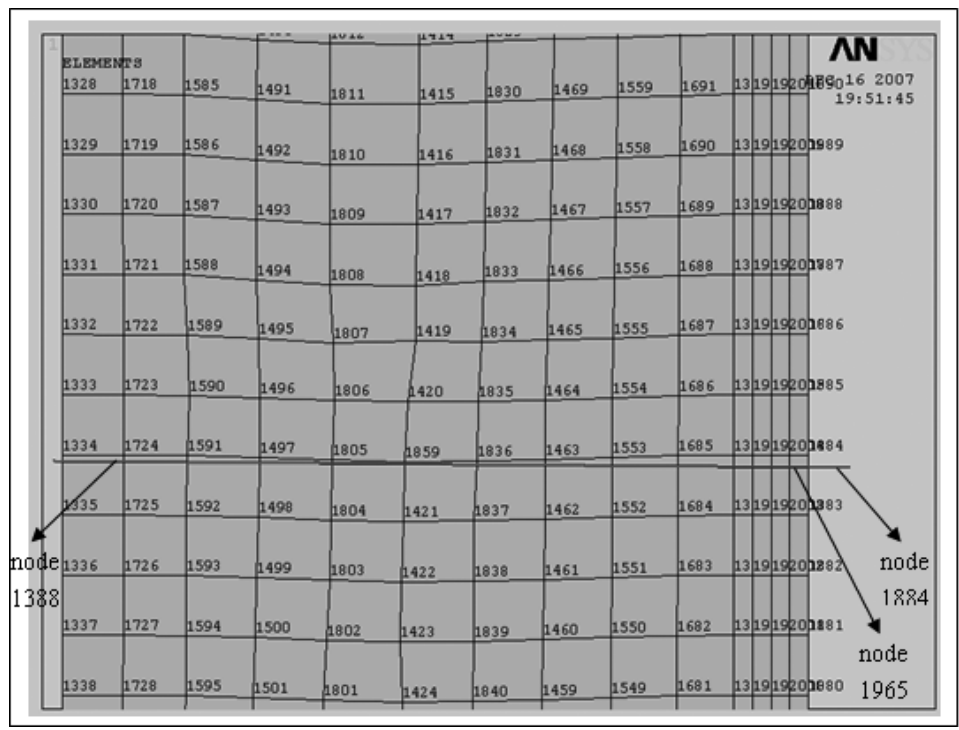

Fig. 18. Enlarged picture of part of nodes of outer shell (red line).

temperature field analysis, the computing time is $25 \mathrm{~ms}$, equivalent to the pressure response time of general artillery guns.

As shown in Figs. 18-20, the temperature of the shell wall under the TBC shelter basically remains at about $20^{\circ} \mathrm{C}$, and the $\mathrm{TBC}$ temperature drops very quickly during the gun pressure response time. Therefore, by taking suitable measures against heat, CPS characteristics are not influenced by the instantaneous high temperature.

For the period from the start of the fire up to the instant the pressure gauge was taken out of the chamber (about $20 \mathrm{~s}$ ), the temperature distribution of the shell is shown in Fig. 21. The outside temperature is much higher, reaching above $100^{\circ} \mathrm{C}$, and the inner 


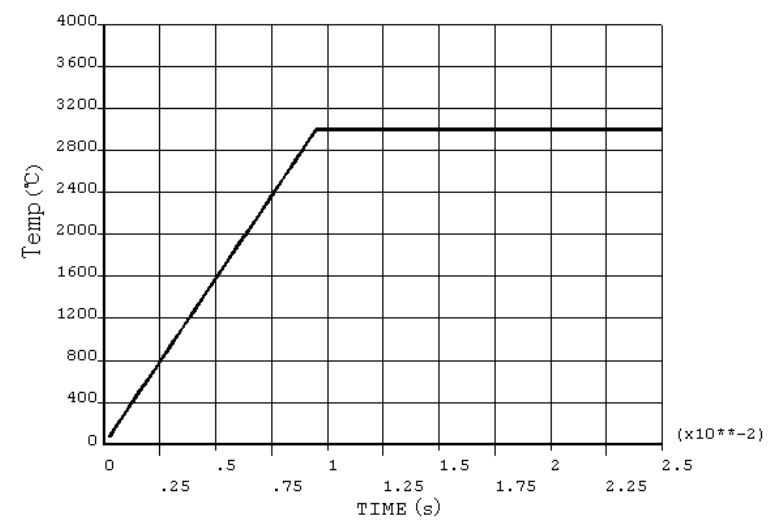

(a)

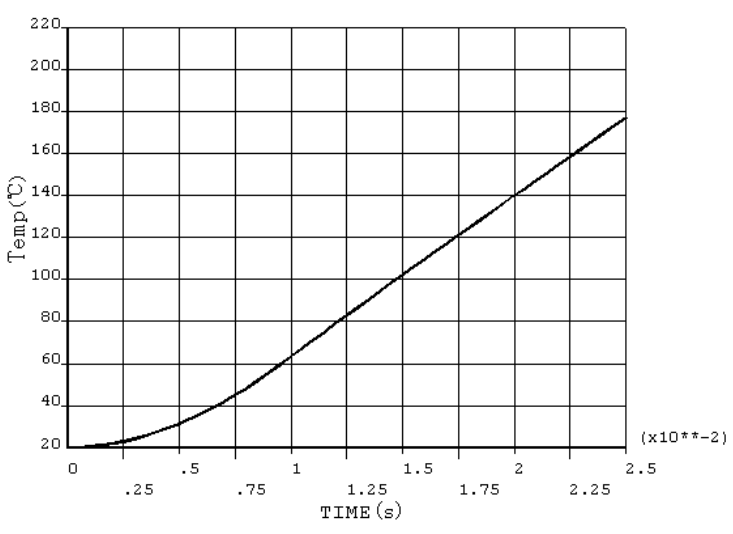

(b)

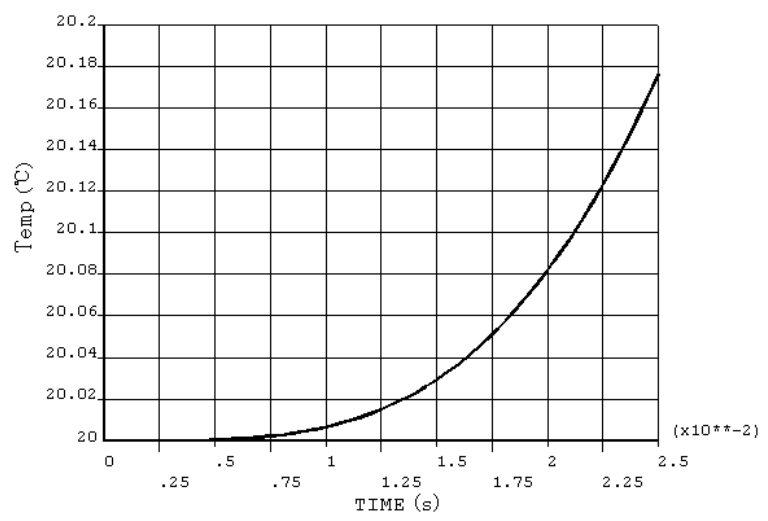

(c)

Fig. 19. Temperature-time chart. (a) Node 1884 (outermost part of thermal coating), (b) Node 1965 (middle thermal barrier coating), and (c) Node 1388 (combining point of outer shell and thermal barrier coating)

temperature remains normal, and the normal operation of the sensor is not affected. The above analysis indicates that the temperature effect on the shell is negligible.

\section{Experimental Results and Discussion}

In order to characterize the capacitance pressure sensors, measurements were carried out. In the experiments, the 1,000 MPa hydraulic calibration machine produced by the Xi' an No. 204 Research Institute was used to measure pressure, and a TH2617 LCR was used to measure capacitance. The experimental result was compared with the theoretical prediction result from a mathematic model. Figure 22 summarizes the simulation 


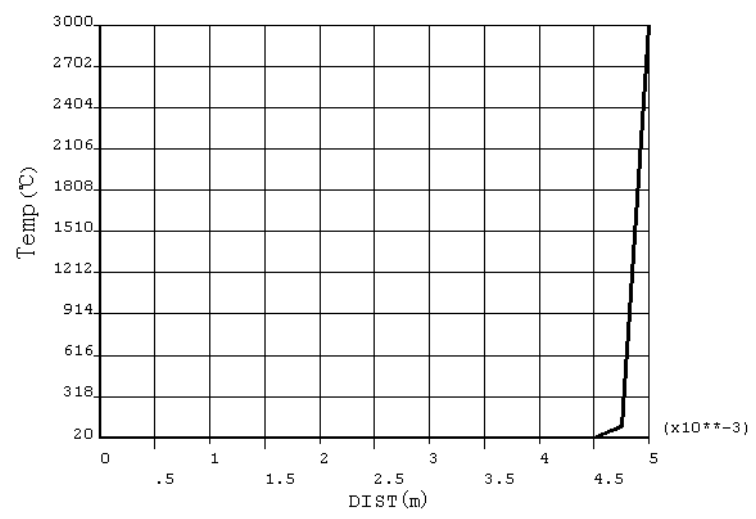

Fig. 20. Temperature distribution along the wall thickness (radius) direction at $25 \mathrm{~ms}$.

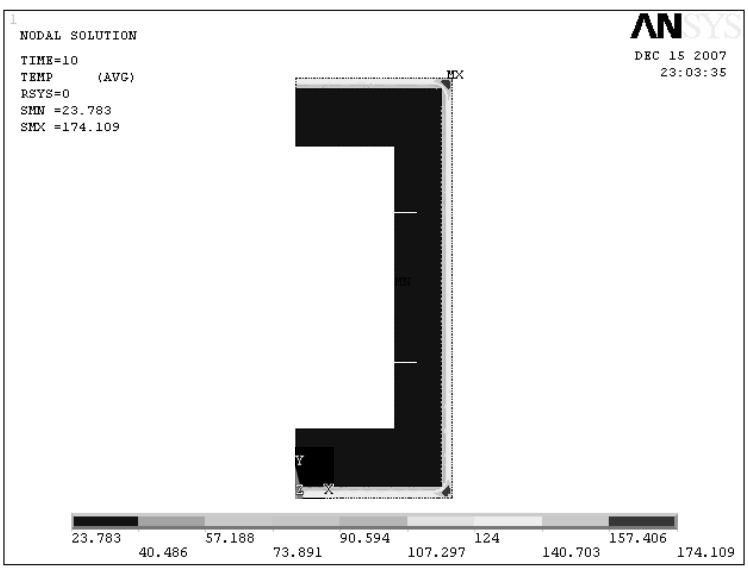

Fig. 21. Temperature distribution of the shell at $20 \mathrm{~s}$.

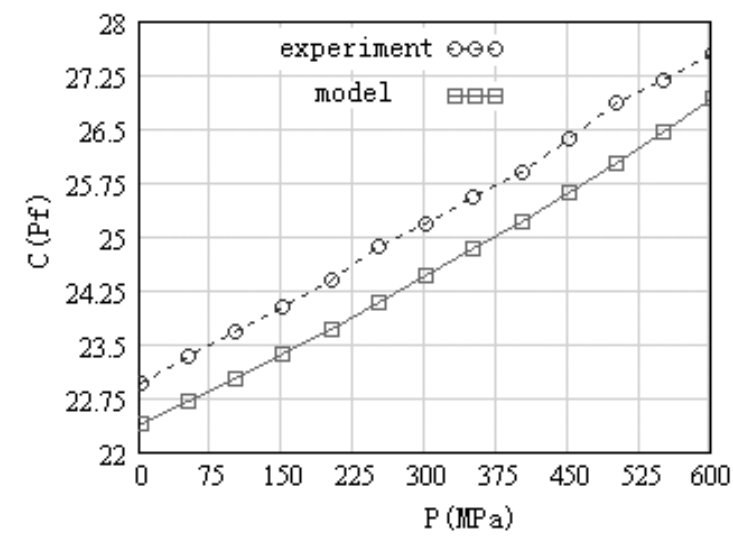

Fig. 22. Relationship between capacitance $C$ and pressure $P$. 
results and experiment results, and it indicates that the experimental results were in good agreement with the simulation results; these results had nearly the same trend. Its sensitivity is $0.00753 \mathrm{Pf} / \mathrm{MPa}$. The maximum working pressure is $600 \mathrm{MPa}$, and from the curve, it follows that the capacitance has an approximately linear relationship with pressure, when the pressure on the sensor is increasing. There is a certain error between the calculated value and the actual value of capacitance measured. The are several reasons for this error: first, there may be interference during the testing; second, there may be some errors in the control of electrode spacing, and third, during the calculation and simulation, some approximations are used and so may introduce system error. By using the compensation and accurate calibration of the processed circuit, these errors could be further reduced so that the output shows a better linear relationship with pressure.

Testing of temperature stability was conducted in a high temperature experimental box. Temperature experiments on the sensors without and with $\mathrm{TBC}$ were carried out

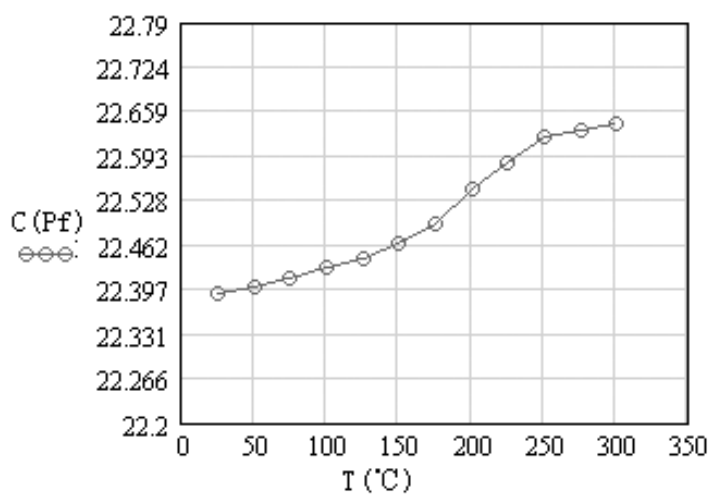

Fig. 23. Capacitance $C$ as a function of temperature $T$ (CPS without TBC).

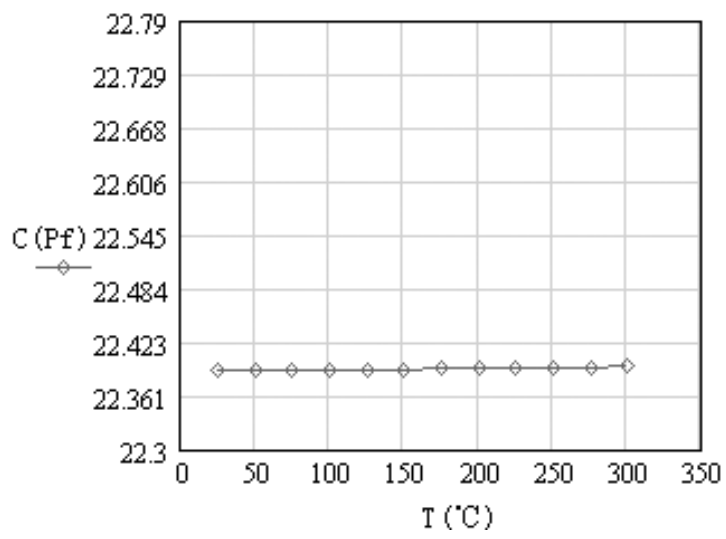

Fig. 24. Capacitance $C$ as a function of temperature $T$ (CPS with TBC). 
under the same experimental conditions. The testing temperatures ranged from room temperature to $300^{\circ} \mathrm{C}$. Two sets of data were obtained. The capacitance-temperature curves tested using the sensors without and with TBC are shown in Figs. 23 and 24, respectively. As shown in Fig. 23, without the coating, capacitance increases linearly and slowly as temperature varies from 25 to $135^{\circ} \mathrm{C}$, and in the range of $135-225^{\circ}$ $\mathrm{C}$, it increases linearly but rapidly. Outside that range, it increases very slowly. As shown in Fig. 24, with the coating, the capacitive value of the CPS hardly changes with temperature change. ${ }^{(14)}$

\section{Conclusions}

The research and development of CPS is presented in this paper. The mechanical and thermal characteristics of the sensor are theoretically simulated using ANSYS. The theoretical results show that it can bear a high pressure of $600 \mathrm{MPa}$, and its inherent frequency up to $52.6 \mathrm{kHz}$ can meet the requirements of the chamber pressure tested. The CPS prototype was further experimentally studied by various tests. The experimental results show that its capacitance is approximately linear with pressure. The sensor possesses a very high stability against temperature. With its stable properties and delicate structure, the sensor can be conveniently used for testing the chamber pressures of various artillery of large, medium, and small calibers. With its many advantages such as the structural simplicity, low cost and small size, the sensor is advantageous for widespread use of IEPG.

\section{Acknowledgements}

This project is sponsored by the foundation of Science and Technology on Electronic Test \& Measurement Laboratory and its serial number is 04011007 . The authors thank Mr. Lang Liu and Mrs. Yan Li for their assistance in the test experiments.

\section{References}

1 Y.-L. Zhang and G.-L. Liu: J. Ballistics 10 (1998) 78.

2 J.-L. Zhang, H.-N. Lu and S.-M. Ren: J. Tai Yuan Institute of Machinery 14 (1993) 22.

3 D.-R. Kong, M.-W. Zhu and Sh.-N. Zhang: Metrology \& Measurement Technique 28 (2001) 15.

4 K. H. L. Chau and S. R. Lewis: Sens. Actuators, A 54 (1996) 472.

5 M. Lemkin and B. E. Boser: IEEE 1996 Custom Integrated Circuits Conf [C], (1996) p. 135.

6 A. Chavan and K. D. Wise: Proc. 9th Int. Conf. Solid-State Sensors and Actuators (Transducers' 97), Chicago, USA, 6 (1997) p.1449.

7 Zh.-L. Hang and Y.-J. Feng: Chinese J. Sens. Actuators 19 (2006) 1807.

8 P. Cheng, Zh. Zhan, D.-D. Li and F. Zhen: Sens. Actuators, A 147 (2008) 672.

9 P. Li and M.-Q. Li: Sensor World 12 (2006) 12.

10 Zh.-W. Wang and L.-J. Wang: J. LiaoNing University Natural Science Edition 32 (2005) 131.

11 S.-P. Chang: Sens. Actuators, A 116 (2004) 198.

12 Z.-Sh. Hang and Sh.-L. Zhou: Elastic mechanics, Hangzhou China, ZheJiang Inustitute Press, 4 (1989) p. 85. 
13 R.-X. Hu and Q.-W. Wang: ANSYS8.2 dsingn for mechanism, China Machine Press, 1 (2005) p. 96.

14 Y. S. Lee and K. D. Wise: IEEE Trans. Electron Devices 29 (1982) 42.

\section{About the Authors}

Xin'e Li was born in Shanxi, China in 1971. She received her BEng degree in Physics from Shanxi Normal University in 1994, and her MEng degree in Testing and Measuring Technology and Instruments from North China Institute of Technology (NCIT) in 2000. Her research is in the field of test, measurement, and sensors.

Jing Zu was born in Hebei, China in 1933. He received his BEng degree from Beijing University of Technology (BIT) in 1954. His research interests are the in field of test and dynamic measurement and related fields.

Tie-Hua Ma was born in Shanxi, China in 1964. He received his BEng degree in Controlling Technology and Instruments from North China Institute of Technology (NCIT) in 1985, his MEng degree in Vibration, Shock and Noise from Nanjing University of Technology (NJUST) in 1990, and his PhD in Precision Instrument and Machinery from Harbin Institute of Technology (HIT) in 1996. He worked at a mobile post doctoral center in armament science and technology in Beijing University of Technology (BIT) from October 1996 to October 1998, and is now working at the Department of Precision Instrument and Mechanology of Tsinghua University. His research interests are the in fields of test and measurement, accelerometer technology and related fields. His email address ismatiehua@nuc.edu.cn

Peng Xu was born in Shanxi, China, on October 17, 1969. He received his Ph.D degree in test Measurement Technology and Instrument in 2006 from North University of China (NUC), China. He is currently a professor at the Science Institute of NUC. Since 2000, his studies have been focused on the high $g$ acceleration test, the reliability of test circuit modulus in high shock and the numerical simulation of structure strength. 